\title{
Pre-designing an electronic card using a multi-domain models ap- proach with DYMOLA
}

\author{
Behrouz Roumizadeh \\ Jean Yves Choley Régis Plateaux \\ Olivia Penas \\ Alain Riviere \\ SUPMECA \\ 3 Rue Fernande Hainaut, Saint Ouen, France \\ behrouz.roumizadeh@supmeca.fr, jean-yves.choley@supmeca.fr, regis.plateaux@ @upmeca.fr, \\ olivia.penas@supmeca.fr, alain.riviere@supmeca.fr
}

\begin{abstract}
This paper presents a new method to model electronic circuits, considering not only the electrical aspect of the circuit but also its geometrical and multiphysical aspects via DYMOLA [1]. The new modeling method based on the representation of the electronic components and their environments, with the multi-domain models. This means combining the geometrical, electrical and multi-physicscal properties in one single mechatronical model. This kind of representation will allow us to simulate the electronic environments regarding their multi-domain aspects and relations. This new method will allows us to perform the pre-dimensioning and pre-placement of the components, through a simple modeling process in DYMOLA environment. We expect the result to be a more rapid pre-dimensioning procedure for the electrical circuits.
\end{abstract}

Keywords: Electrical circuit; multi-physics; multi domain modeling; DYMOLA; mechatronics.

\section{Introduction}

The design of automotive electrical circuits is a complicated procedure, which includes vast parts of science, from the pure electrical Eng., to the multiphysical simulations such as thermal simulations. This will result in the need for different and mostly unrelated modeling and simulation programs, in order to design and optimize the conception of electrical components. The result would be a complete mechatornic problem which includes electrical and mechanical problems. It is obvious that unrelativity of the different simulations, will result in the increase of the time and cost of the design and design process.
Generally, design of an electrical circuit begins with its schematic model (logical view). This stage of design consists of the electrical and logical connections between the components, and can be modeled and simulated by the different software such as CADENCE [2], ZUKEN [3], and the electrical library of DYMOLA.

Simulation of the electrical schematics will result in the electrical properties of the components and connections, which are needed for the multi-physical simulations. Although the electrical simulations at this level can be performed without taking into consideration the geometrical aspects of the circuit and its environment, but once the multi-physical simulations have been carried out, the circuit has to be remodeled and re-simulated due to changes originating from the electrical-multi-physical interactions.

Once the logical aspect of the circuit has been validated, components have to be placed on the surface on which they will operate. This step will be followed by a routing procedure.

Existing electrical software is only capable of performing the component placement and routing of the PCBs (Printed Circuit Boards) on the 2D surfaces [4]. There is also some new software [5] [6] and algorithms [4][7] which offer this possibility on the 3D surfaces, although existing software does not offer any solutions for some other electrical designs such as high current electronics and power electronics, for which the 3D MCAD(Mechanical CAD) systems are used as a design system.

The present design cycle is presented in fig.1. Once the electronic card has been designed, the multiphysical simulations, which are mostly the FE simulations, will be performed to validate the operability of the circuit, from the multi-physical point of view. 


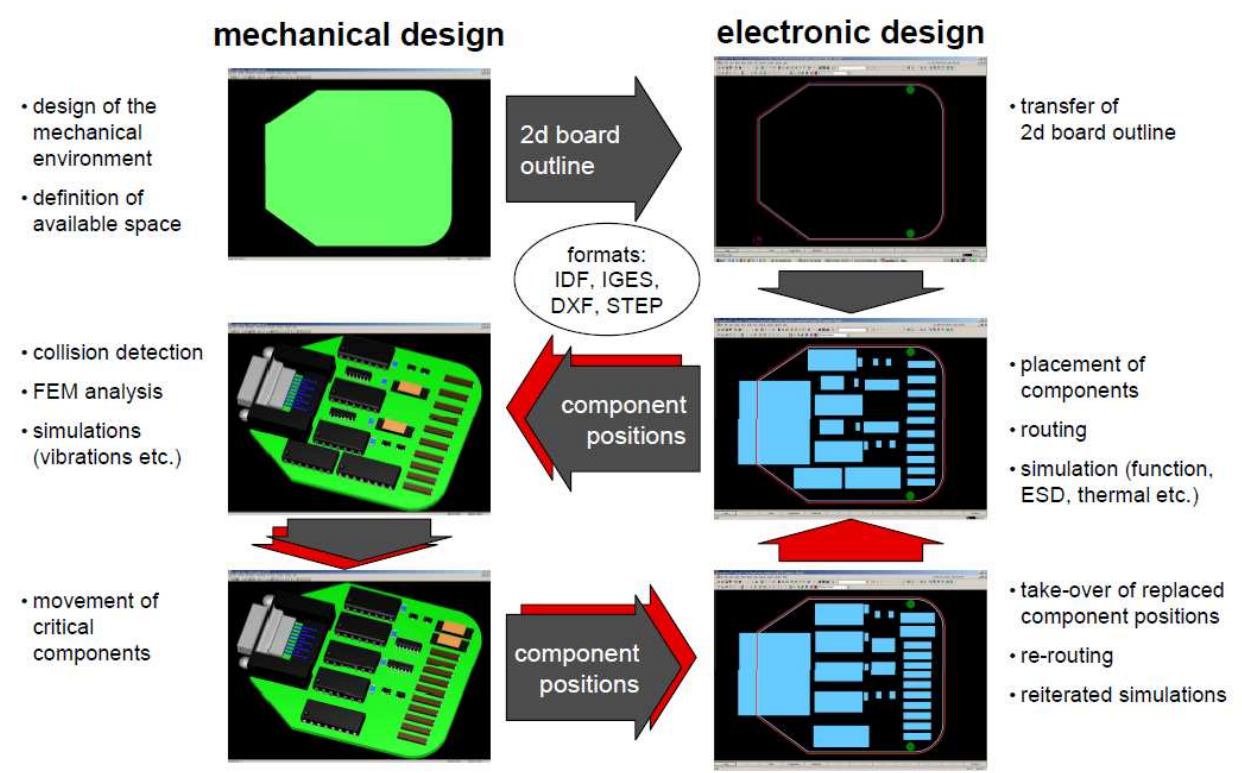

Figure 1: the actual electronic design cycle [8]

The two most important multi-physical simulations for electronics are the thermal and EMC (Electro Magnetic Compatibility) simulations. The circuit has to be simulated within its environments, from the thermal [9] and EMC [10] point of view, so that its functionality will be validated. As can be observed from the figure above, the position of the components may be changed once the thermal simulations for instance have been performed, and the board must be rerouted. It is obvious that this cycle and specially, the FE simulations are time consuming and costly specially because this conception cycle will continued till the optimized circuit would be achieved.

A solution for this problem would be a rapid prepositioning and pre-sizing procedure, which would result in a semi-optimized electronic card. We believe that this will reduce the need of FE simulations. This simulation process has to combine some multidomain properties in one single model. DYMOLA proposes a good graphical environment which enables us to perform this multi-domain modeling.

As has been said above, DYMOLA proposes the ability to simulate electrical circuits and there are existing libraries to do so. It also proposes thermal models which enables thermal simulations, although a linked electro-thermal simulation which takes into account geometrical properties of the components and their placements, does not exist. To do so the designer has to calculate the geometrical effects on the thermal properties and with them perform the thermal simulations. Our proposition is to introduce a new modeling method so that it includes electrical, geometrical and thermal properties in a single model so that they are related. The representation of the geometrical and multi-physical properties such as thermal properties is simplified, so that the simulation will be performed rapidly and it will serve as a pre-dimensioning process so that it will reduce the need for the FE simulation and replacement cycle.

\section{Mathematical multi-domain para- meters relations}

To perform the new modeling procedure we will need to have the mathematical relations of the different multi-physical properties, electrical properties and the geometrical properties with each other so that we could build the new multi domain models in DYMOLA. This is more essential in the sections that the different properties have the interactions with each other, such as the heating resistances or the surfaces which the multi-physical properties are related to the geometrical and electrical properties. [11] and [12] propose a method to extract such relations with the help of the topological diagrams. The capability of this has been shown by [13] which explained the need of the topological structure to model the complex systems. Fig. 2 shows the algebraic diagram which by that we can associate the different properties of our model. On the first step the analysis will start from nodes on the Primal object. The nodes present the properties of the nodes which in our case are the coordination of the nodes $(\mathrm{P})$, electrical potential of the nodes $(\mathrm{V})$ and the temperature of each node $(\mathrm{T})$. We will call the matrix which contains these properties matrix $[\mathrm{N}]$. The incidence matrix [C], which builds up of the relations between different nodes will capable us to have the relations of the 
same properties between two nodes(Branches) such as the vector between two nodes, the potential differences between nodes and the temperature differences., this will be presented in matrix [B]. The dual object $R_{B}$ for us will be the current and the heat flow in the branches. To arrive to the $R_{B}$ we would need the admittance matrix $Y_{B}$ which represents the relation between different properties.

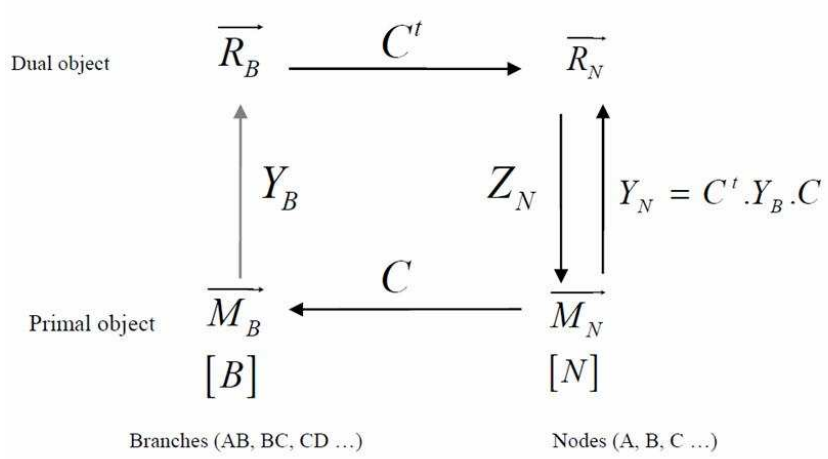

Figure 2: algebraic diagram of the relations between different properties of the model

As it can be seen, once we have the topological diagram of the model, from any property as the departure data we will be capable of calculating the other properties of the model. These relations will help us to perform our modeling and analysis. Although this method will respond to our need of knowing the relation between the components in the model but it would be hard to perform if the model is complicated. [14] had showed that by partitioning the matrices into smaller model and matrices we will be able to simplify the model. This is done by associate to each part of the model a topological diagram and incidence matrix $[\mathrm{C}]$ and by connecting the smaller parts together construct the bigger incidence matrix. The possibility of partitioning the incidence matrix into smaller parts of the model will permit us to easily extract the equations of the models which do not exist in the standard DYMOLA library. As an example the relations of the multi-physical properties of a resistor are already exist in DYMOLA, and we just need to connect the existing multi domain models together by a new built connector which can support all the parameters of these models, but as the thermal relations on a surface with their interactions with the geometry does not exist, the relations between the different parameters of this new model has to be extracted by this method.

To calculate the thermal properties we have included the thermal resistance concept [15]. This will reduce the variables which are all included in the thermal resistance $\mathrm{R}_{\mathrm{th}}$ and facilitate the calculations. Algebraic diagram in fig. 3 present the different variables in our calculations and their relations together. For the reason that the entire variable has to be calculated in the same time with the different interactions that they have with each other the relation between the variables which will be obtained by this diagram is quit complicated.

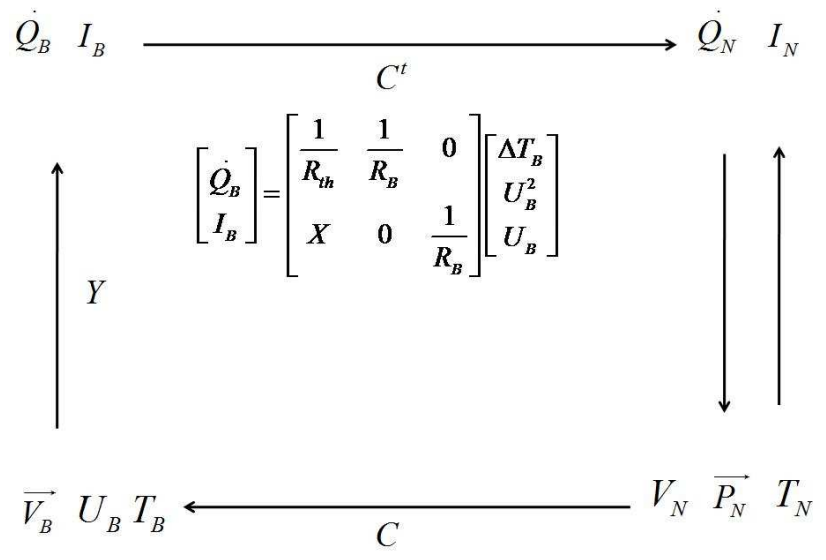

Figure 3: multi-physical parameters relations

To reduce the complicity of the equations [12] offers a possibility of separating the diagram in more simple diagrams, solve these simpler diagrams and then calculate their interactions' with each other.

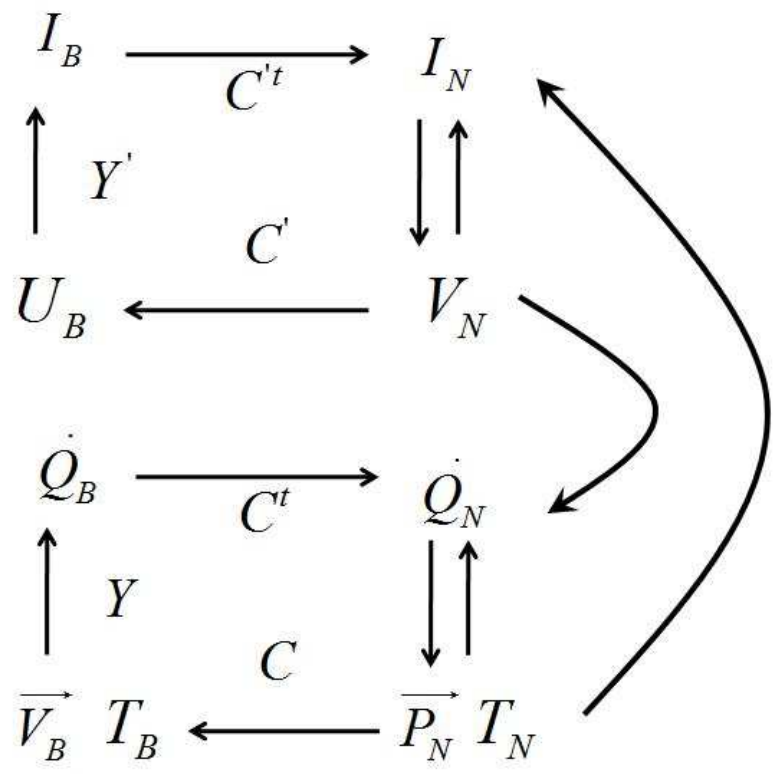

Figure 4: electrical and thermal algebraic diagrams and their interactions

This method will reduce the complexity of the equations and has the advantage that as the upper section (electrical diagram) which represent the electrical equations already exist in DYMOLA, it will give us the relation of these existing models with the newer parameters needed in the models. 


\subsection{Thermal characteristics of the electrical boards}

In the electrical boards and circuits most of the heat passes through the board or surface of the circuit. This produces a need to include the heat transfer from the surfaces in the simulation. In the DYMOLA thermal library, there is no mean to calculate the heat transfer on a $2 \mathrm{D}$ surface. We have introduced two plane models with the ability to calculate the heat transfer between the connectors' positions in relation to the position of the connectors.

The first option to calculate the thermal properties on the plane and between different points which will be represented by the connectors, we will use the concept of the radial heat conduction on a disc [15] fig.5.

$$
R_{t h}=\frac{\ln \left(r_{2} / r_{1}\right)}{2 \pi L \lambda}
$$

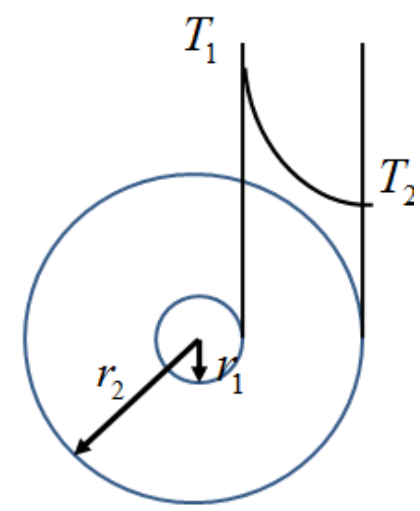

Figure 5: thermal resistance in a radial conduction on a plane

Although this method is simple to calculate and use, it would be applicable only if the surface could be supposed unlimited due to the dimensions of the $r_{1}$ and $\mathrm{r}_{2}$. The other option is to use the equivalent electrical network [16] fig.6, which represent the system with the system with the thermal resistances and capacitors which can be simulated as an electrical network.

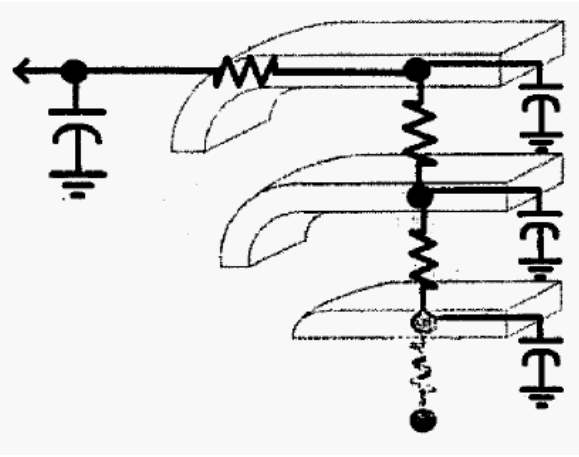

Figure 6: equivalent electrical network representation of a thermal system

To simplify the models in the first stage of modeling procedure we suppose the problem as a steady state situation so that we can neglect the $\mathrm{C}$. This will simplify our plane thermal model to a model which has been divided by the smaller cubes which each will be represented by their thermal resistances. Fig. 7

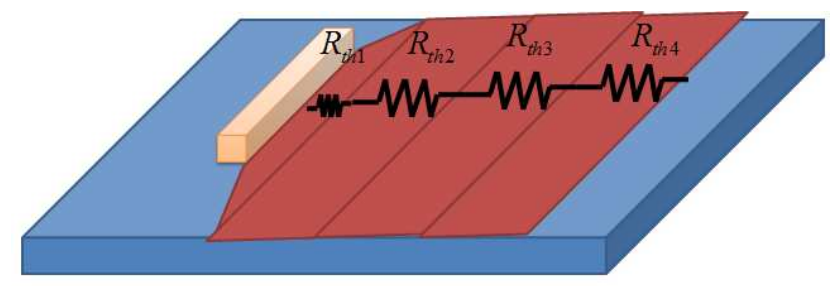

Figure 7: plane thermal model represented by the thermal resistance network concept

These two thermal models can be used for modeling the plane model including its geometrical equations in DYMOLA.

\section{Modeling procedure}

Once the mathematical equations and relations of the models have been extracted the models and the modeling procedure can be introduced. This new modeling method will need a new library which includes the electrical components presented with the new concept, and new components which will serve as the environment of the electronic circuit or the surfaces on which it will be installed. All of these new models ought to include the multi-domain models, properties and connectors inside them. Fig 8 shows a new internal representation for a resistance component.

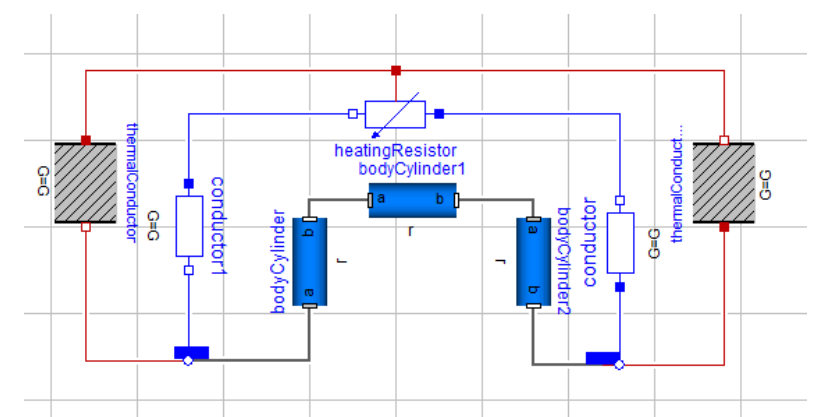

Figure 8: Internal multi-domain representation of an electrical resistance

It can be observed that the new model not only represents the electrical properties of the resistance but also its geometrical and thermal properties. This new model has been introduced by utilization of the existing DYMOLA library models with connecting them to a new connector. This new connector contains the combined properties needed in this model. This connector is needed to ensure the multi-domain 
modeling within a single model. Fig 9 shows the properties of the new connector used to connect new models build in DYMOLA.

\begin{tabular}{|l|l|l|}
\hline Type & Name & Description \\
\hline Real & $\mathrm{n}[3]$ & the normal vector \\
\hline Position & $\mathrm{P}[3]$ & position [m] \\
\hline Temp K & T & tempreture of the connection $[\mathrm{K}]$ \\
\hline flow HeatFlowRate & Q_flow & heat flow pass by the connection [W] \\
\hline flow Current & i & current pass by the connection $[\mathrm{A}]$ \\
\hline Voltage & $\mathrm{v}$ & the votltage at the connection $[\mathrm{V}]$ \\
\hline
\end{tabular}

Figure 9: New connector characteristics

It is obvious that due to the need of taking into account the newer properties in the system such as the electromagnetic properties, other variables have to be introduced in the connector.

To perform the multi-physics simulations, we also need to integrate the environments of the circuits. To do so, we propose new geometrical models, such as plane model which is presented in fig 10 , so that the other components can be installed on them, and they themselves can be connected to another environmental model.

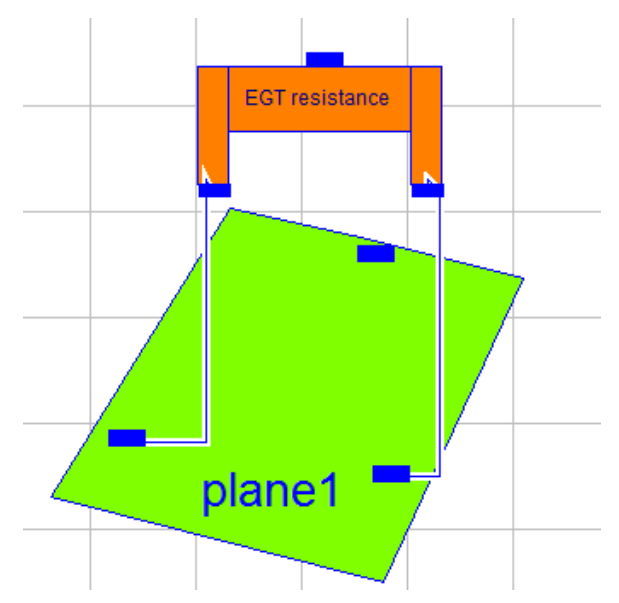

Figure 10: integration of the electrical components in their multidomain environment e.g. plane

As the components are placed on the different places on the plane, we need to be capable of calculating the temperature $(\mathrm{T})$, and the thermal flow $(\mathrm{Q})$ between the different connector points. As it has been explained in section two, this is possible by using the thermal resistance concept. As the thermal resistance is related to the geometry, and the heat produced is related to the electrical properties of the circuit, the relations which would be achieved by the procedures in the section two would be used to build the new multi-domain models such as the plane model.
Using the multi-domain electrical and geometrical models to model the electrical circuits which are made by this method, we are be able to simulate the electrical circuits in DYMOLA not only due to their electrical properties but also geometrical and thermal properties in one single stage. The advantage would be that normally, the circuit has to be modeled and simulated due to one of these properties (electrical, geometrical and thermal) and the relations between each simulation has to be performed by the paperwork. For instance to perform the thermal simulations the electrical and geometrical variables of the circuits has to be transferred by hand from one simulation to the other.

\section{Conclusion}

We offer a new method to model and simulate electrical circuits, considering their geometrical and multi-physical properties in one single model. This new method will result in a pre-optimized electrical design and components placement, which will reduce the need of costly Finite Element simulations.

The possibility of exchange the geometrical properties with CATIA [17] will serves as a strong positive point for this new modeling method because the geometrical data and positioning of the components can be used in modeling a detailed geometry of the electrical circuits with CATIA. This and a procedure to perform an electrical routing on the $3 \mathrm{D}$ surfaces with the help of DYMOLA and CATIA would be subject of the future work.

\section{References}

[1] http://www.dynasim.se

[2] http://www.cadence.com

[3] http://www.zuken.com

[4] K. Feldmann, Y. Zhuo, C Alvarez, "3D Gridless routing for the design of Molded Interconnect Devices (MID)" Production engineering, XII-2 89-94. 2005.

[5] NEXTRA http://www.mecadtron.de

[6] $E^{3}$-SeriesZUKEN http://www.zuken.com/products/e3series.aspX

[7] Y Zhuo, C. Alvarez, K. Feldmann, "An Integrated design system for Molded Interconnect Devices(3D-MID)", Digital enterprise technology, 2007 
[8] T. Krebs, "3D mechanical CAD collision detection with Allegro PCB editor and NEXTRA", proceedings of EMEA conference, May 2007

[9] FLOTHERM http://www.mentor.com/products/mechanical /products/flotherm

[10] Q3D

http://www.ansoft.com/products/si/q3d_extra ctor/

[11] Franklin H. Branin Jr. "The AlgebraicTopological Basis for network analogies and the vector calculus." Symposium on generalized networks. 04/1966.

[12] Bjorke O., "Manufacturing systems theory", 1995.

[13] R., Penas O., Riviere A., Choley J.Y., "A need for the definition of a topological structure for the complex systems modeling"., CPI2007.

[14] Elmqvist H., "A structured model language for large continuous systems". PhD theses. LIT 1978.

[15] G.F. Hewitt, G. L. Shires and T. R. Bott, "Process heat transfer", CRC press, 1993.

[16] J.M. Ortizo-Rodriguez, D. Berning, M. Hernandez, "Lumped-parameter Thermal Modeling of an IPEM using Thermal Component Models" IEEE 2004

[17] P. Bhattacharya, N. S. Welakwe and R. Makanaboyina, "Integration of CATIA with Modelica", proceedings of Modelica conference, 2006 